\title{
A urine biomarker for severe obstructive sleep apnoea patients: lipocalin-type prostaglandin D synthase
}

\author{
Yuichi Chihara', Kazuo Chin², Kosuke Aritake, Yuka Harada', Yoshiro Toyama1, \\ Kimihiko Murase ${ }^{1}$, Chikara Yoshimura², Takefumi Hitomi², Toru Oga², \\ Michiaki Mishima' and Yoshihiro Urade ${ }^{3}$
}

\begin{abstract}
Affiliations: ${ }^{1}$ Dept of Respiratory Medicine, Graduate School of Medicine, Kyoto University, Kyoto, ${ }^{2}$ Depts of Respiratory Care and Sleep Control Medicine, Graduate School of Medicine, Kyoto University, Kyoto, and ${ }^{3}$ Depts of Molecular and Behavioral Biology, Osaka Bioscience Institute, Osaka, Japan.
\end{abstract}

Correspondence: K. Chin, Depts of Respiratory Care and Sleep Control Medicine, Graduate School of Medicine, Kyoto University, 54 Shogoin, Kawahara-cho, Sakyo-Ku, Kyoto 606-8507, Japan.

E-mail: chinkakuhp.kyoto-u.ac.jp

ABSTRACT Lipocalin-type prostaglandin D synthase (L-PGDS), which is responsible for the biosynthesis of prostaglandin $\mathrm{D}_{2}$, has been reported to have a close connection with cardiovascular disease and sleep regulation. This study aimed to test the hypothesis that the L-PGDS level is a useful marker to identify patients with obstructive sleep apnoea.

64 subjects were enrolled in this prospective study. Urinary concentrations of L-PGDS were measured in the morning. Measurements were made every $4 \mathrm{~h}$ in 25 of the 64 patients. Endothelial function was assessed by the reactive hyperaemia peripheral arterial tone index.

Circadian variations in L-PGDS concentrations had a significant time-dependent fluctuation $(\mathrm{p}=0.0002)$. L-PGDS was higher in the subjects with severe obstructive sleep apnoea (median $784.7 \mathrm{ng}$ per mg of creatinine, $n=23$ ) than in control subjects (262.1 ng per mg of creatinine, $n=16 ; p=0.004)$ and in those with moderate obstructive sleep apnoea (371.7 ng per mg of creatinine, $n=25 ; \mathrm{p}=0.0008$ ). After 2 days of continuous positive airway pressure treatment, L-PGDS concentrations in severe obstructive sleep apnoea subjects $(n=12)$ decreased significantly $(p=0.02)$ to levels present in control subjects whereas endothelial function did not change significantly. Morning urinary L-PGDS concentrations had significant correlations with the apnoea/hypopnoea index $\left(\mathrm{R}^{2}=13.9 \%\right)$ and serum high-density lipoprotein cholesterol $\left(\mathrm{R}^{2}=6.2 \%\right)$, but not with sleepiness.

Urinary L-PGDS might be a moderately useful marker to identify patients with severe obstructive sleep apnoea.

@ERSpublications

Urinary lipocalin-type prostaglandin D synthase might be a moderately useful marker to identify patients with severe OSA http://ow.ly/pBuac

This article has supplementary material available from www.erj.ersjournals.com

Received: Aug 252012 | Accepted after revision: Nov 182012 | First published online: Dec 062012

Clinical trial: This study is registered at www.clinicaltrials.gov with identifier number NCT01096433.

Support statement: This work was supported by grants from the Japanese Ministry of Education, Culture, Sports, Science and Technology (grant numbers 22590860, 22249031, 23659109 and 24621005), Respiratory Failure Research Group and Health Science Research Grants (Comprehensive Research on Life-Style Related Diseases including Cardiovascular Diseases and Diabetes Mellitus) from the Ministry of Health, Labor and Welfare of Japan, and the Japan Vascular Disease Research Foundation.

Conflict of interest: Disclosures can be found alongside the online version of this article at www.erj.ersjournals.com

Copyright @ERS 2013 


\section{Introduction}

Obstructive sleep apnoea (OSA) is one of the most important medical conditions identified in the last 50 years and is a major cause of morbidity and mortality throughout the world [1].

Most patients with OSA that should be treated are undiagnosed. One reason is that the diagnostic methods for OSA, such as polysomnography (PSG), are difficult to perform. Therefore, a reasonable biomarker for OSA would be extremely helpful, especially in identifying patients who have OSA with a degree of severity that would put them at risk for cardiovascular disease (CVD).

Prostaglandin $(\mathrm{PG}) \mathrm{D}_{2}$ is formed by the action of PGD synthases on the cyclooxygenase (COX) product $\mathrm{PGH}_{2}$. $\mathrm{PGD}_{2}$ is widely distributed in rat and human brain [2]. In peripheral tissues, $\mathrm{PGD}_{2}$ executes a wide range of functions, including vasodilatation, inhibition of platelet aggregation, glycogenolysis, vasoconstriction, allergic reaction mediation and intraocular pressure reduction [3-8]. In the brain, $\mathrm{PGD}_{2}$ has been shown to contribute to sleep induction, modulation of body temperature, olfactory function, hormone release, nociception and neuromodulation [9-12]. Thus, because $\mathrm{PGD}_{2}$ has significant effects on platelet aggregation, vasodilation and vasoconstriction, it has been supposed that $\mathrm{PGD}_{2}$ is relevant to the occurrence of CVD. Although the prostanoids, including $\mathrm{PGD}_{2}$, are released from cells immediately after synthesis, it is believed that prostanoids work only locally, near their site of production, because they are either chemically or metabolically unstable [13].

One of the enzymes characterised as a PGD synthase, which catalyses the isomerisation of $\mathrm{PGH}_{2}$ to $\mathrm{PGD}_{2}$, is the lipocalin-type prostaglandin D synthase (L-PGDS) [14], and is responsible for the biosynthesis of $\mathrm{PGD}_{2}$ in the brain and heart (cardiovascular system). L-PGDS is a unique protein with enzyme activity and ligandbinding properties. L-PGDS binds various lipophilic compounds, such as retinoids, bilirubin, biliverdin, gangliosides and amyloid- $\beta$ peptides, with high affinity, acting as an extracellular transporter of these compounds and serving as an endogenous amyloid- $\beta$ chaperone to prevent amyloid deposition in vivo [15]. The half-life of L-PGDS in canines was reported to be $0.77 \mathrm{~h} \mathrm{[16].}$

L-PGDS was confirmed to be secreted into blood and urine [14], and we have established a system to measure its urine, serum or plasma levels by an ELISA system [14, 17-19]. L-PGDS is a very stable enzyme and is highly resistant to heat treatment [14] and protease digestion [20], whereas $\mathrm{PGD}_{2}$ is an unstable substance, as mentioned earlier [13]. L-PGDS is localised where $\mathrm{PGD}_{2}$ would have its effect, that is, in the central nervous system, male genital organs and the human heart. In the human heart, L-PGDS is localised in myocardial and atrial endocardial cells, smooth muscle cells in the arteriosclerotic intima, and in the atherosclerotic plaques of severely stenotic coronary arteries. In addition, the chemical properties of L-PGDS are similar to those of serum albumin; however, its molecular weight is much smaller than that of serum albumin (26000 versus $66000 \mathrm{Da})$. Thus, L-PGDS more easily passes through glomerular capillary walls of the kidney than serum albumin. Indeed, it has been reported that urinary L-PGDS excretion increased in the microalbuminuric stage in patients with type 2 diabetes mellitus (DM) and in hypertensive patients who were apparently free from overt proteinuria [21, 22]. Although the enzymatic activity of L-PGDS cannot be determined, the amount of serum or urinary L-PGDS increased when conditions such as coronary heart disease, hypertension or type 2 DM worsened [21-23].

As OSA induces multi-organ damage and diseases such as hypertension, DM, renal insufficiency, coronary disease and cerebral-cardiovascular diseases, the degree of severity of OSA in individuals with OSA would have significant associations with morbidity and mortality from these conditions [24]. In addition, serum L-PGDS levels were slightly elevated in individuals with OSA with excessive daytime sleepiness [25]. Thus, we hypothesised that L-PGDS could be a biomarker for OSA because of its close relationships with sleep and CVD. In consideration of this hypothesis, we tested whether plasma or urine L-PGDS would be a powerful biomarker for OSA.

\section{Methods}

Study subjects

64 clinically stable adults (age $>20$ years) with suspected OSA were consecutively enrolled in the present prospective study. This study is registered at www.clinicaltrials.gov with identifier number NCT01096433.

Major exclusion criteria were history of CVD, DM under treatment with hypoglycaemic agents or insulin, use of glucocorticoid or nonsteroidal anti-inflammatory drugs, and being a current smoker. This study was approved by the Ethics Committee of Kyoto University (Kyoto, Japan). All patients gave written informed consent to participate. The other exclusion criteria are shown, in detail, in the online supplementary material. 


\section{Study design}

At baseline, the subjects' medical history was recorded and a physical examination was performed. In the medical history, hypertension was defined as a systolic blood pressure $\geqslant 140 \mathrm{mmHg}$ or diastolic blood pressure $\geqslant 90 \mathrm{mmHg}$, or use of an antihypertensive medication. Dyslipidaemia was defined as serum lowdensity lipoprotein cholesterol $\geqslant 140 \mathrm{mg} \cdot \mathrm{dL}^{-1}$ or high-density lipoprotein cholesterol (HDL-C) $<40 \mathrm{mg} \cdot \mathrm{dL}^{-1}$, triglycerides $\geqslant 150 \mathrm{mg} \cdot \mathrm{dL}^{-1}[26]$, or use of an antilipidaemic medication.

All patients underwent attended diagnostic overnight PSG. Before the patient slept, urine samples were collected at 22:00 h. In addition, the first urine voided in the morning following the overnight PSG was collected at 06:00 h. Peripheral venous blood samples were collected from 06:00 h to 7:00 h following a 12-h overnight fast and PSG. Morning endothelial dysfunction measured by reactive hyperaemia peripheral arterial tone (RH-PAT) [27] was measured. After the overnight PSG, five blood pressure measurements, each 1 min apart, were taken in the morning after the patient had rested for at least $5 \mathrm{~min}$ in the supine position. The average of the latter two readings was calculated.

After 2 days of continuous positive airway pressure (CPAP) treatment, urine and blood samples were collected at the same time as during and after PSG. In all of the patients, adequate CPAP pressure was determined to have overcome obstruction and all flow limitations by a full night's titration.

During the PSG day, we investigated the circadian variations in urinary L-PGDS concentrations in 25 patients who were randomly selected from all 64 subjects at 4 -h intervals except for midnight (14:00, 18:00, 22:00, 06:00 and 10:00 h). Urinary sampling was not performed at midnight in order not to disturb physiological sleep.

\section{Polysomnography}

PSG was performed according to recommendations in American Academy of Sleep Medicine manual (online supplementary material). Apnoea/hypopnoea index (AHI) values were expressed as the number of episodes of apnoea and hypopnoea per hour over the total sleep time. OSA severity was defined by the AHI as follows: control (AHI $<15$ events $\left.\cdot h^{-1}\right)$, moderate OSA $\left(15 \leqslant \mathrm{AHI}<30\right.$ events $\left.\cdot \mathrm{h}^{-1}\right)$ and severe OSA (AHI $\geqslant 30$ events $\left.\cdot h^{-1}\right)$.

\section{Measurements of plasma and urinary L-PGDS concentrations}

In the present study, plasma samples were centrifuged immediately at $1470 \times g$ at $4^{\circ} \mathrm{C}$ for 15 min and urine samples were pooled (not centrifuged), as described previously [15, 17-19]. The separated samples were stored at $-80^{\circ} \mathrm{C}$ until assay. Concentrations of urinary or plasma L-PGDS were measured by an ELISA using two monoclonal antibodies, Mab-7F5 and Mab-1B7, as described previously (online supplementary material) [15, 17-19].

With this ELISA system, it has been demonstrated that intra- and interassay coefficients of variation in urine samples ranged from $3.2 \%$ to $5.8 \%$ and $7.6 \%$ to $8.3 \%$, respectively. The intra- and interassay coefficients of variation in serum samples were $3.6 \%$ and $5.8 \%$, respectively. The ELISA showed no significant interference by a variety of urinary constituents [18]. In addition, it was shown that serum and plasma L-PGDS values in individual subjects were almost the same [15]. All the samples were measured in duplicate and the results were averaged.

\section{Other parameters}

Venous blood samples were taken in the fasting state in the morning after one night of PSG and examined for markers of glucose and lipid metabolism and C-reactive protein. As it was difficult to measure urinary catecholamine and L-PGDS levels at the same time, we measured plasma catecholamine levels.

\section{Measurements of the RH-PAT}

The RH-PAT is a newly established method to measure endothelial function [27]. Endothelial dysfunction as measured by RH-PAT has been reported in patients with OSA [28]. Morning endothelial function assessed by a finger plethysmographic device (Itamar Medical Ltd., Caesarea, Israel) that allows the isolated detection of pulsatile arterial volume changes [27] was measured after an overnight PSG and after 2 days of CPAP.

\section{Statistical analysis}

Data were analysed using JMP 9.0 (SAS Institute, Inc., Cary, NC, USA). Continuous variables were expressed as mean \pm SE or median values and ranges because the sample size of each group was small. The associations between patients' characteristics, PSG data, biomarkers (blood and urine) and OSA severity were assessed by the Kruskal-Wallis test. When a significant difference was observed, we used the Bonferroni-corrected t-test to identify where differences were significant. We evaluated the sensitivity and 
specificity of the cut-off value of L-PGDS for predicting severe OSA with the use of receiver operating characteristic curve analysis, estimating the area under the curve (AUC). The relationship between L-PGDS concentrations (urine and plasma), the RH-PAT index and other parameters were analysed by Pearson's correlation coefficient test. Multiple regression analysis was performed to adjust for confounders such as age, sex, body mass index (BMI), and morning systolic and diastolic blood pressure. Next, multiple regression analyses, with a p-value $<0.10$ required for entry into the models, were performed to identify those variables that could best predict morning urinary L-PGDS.

To investigate changes in L-PGDS concentrations, the RH-PAT index, and other parameters before and after 2 days of CPAP, comparisons of data between those two time-points were tested by a paired t-test. Multiple ANOVA with repeated measures was performed to analyse urinary L-PGDS concentrations across the 24 -h period. In all analyses, p-value $<0.05$ was considered statistically significant.

\section{Results}

Clinical characteristics of study subjects, L-PGDS concentration and RH-PAT index according to OSA severity

Patient characteristics, PSG data and laboratory data are shown in table 1. There were significant differences among the groups in morning urinary L-PGDS concentrations $(p=0.0009)$ but not in night urinary L-PGDS concentrations $(p=0.19)$ and plasma L-PGDS levels $(p=0.09)$ (table 1 and fig. 1a). After adjustment for age and BMI, subjects with severe OSA had significantly higher morning urinary L-PGDS values than control subjects $(\mathrm{p}=0.007)$ and subjects with moderate OSA $(\mathrm{p}=0.002)$. There were significant differences among the groups in the RH-PAT index (fig. 1b).

The cut-off value for predicting severe OSA with minimal false-negative and false-positive errors was $621.8 \mathrm{ng} \cdot \mathrm{mg}^{-1}$ of creatinine (sensitivity $65.2 \%$, specificity, $85.4 \%$ ). This cut-off value had moderate accuracy for predicting severe OSA (AUC 0.78) (fig. 2).

\section{Relationship between urinary L-PGDS concentrations, RH-PAT index and clinical indices}

Morning urinary L-PGDS concentrations were positively correlated with several parameters, including the AHI (table 2 and fig. 3). Morning urinary L-PGDS concentrations were positively correlated with AHI after adjustment for age, sex, BMI and morning systolic and diastolic blood pressure (fig. 3). There was a strongly positive correlation between morning and night urinary L-PGDS concentrations $(p<0.0001)$. The Epworth Sleepiness Scale did not correlate significantly with morning urinary L-PGDS (table 2).

After adjustment for age, sex, BMI, and morning systolic and diastolic blood pressure, morning urinary L-PGDS concentrations were still positively correlated with the AHI $(\beta$ coefficient $=0.373 ; p=0.006), 3 \%$ oxygen desaturation index (ODI) $(\beta=0.322 ; p=0.02)$, arousal index $(\beta=0.370 ; p=0.007)$ and plasma noradrenaline $(\beta=0.258 ; p=0.04)$. The RH-PAT index was negatively correlated with the AHI $(\beta=-0.305$; $\mathrm{p}=0.04)$ and arousal index $(\beta=-0.359 ; \mathrm{p}=0.01)$ after adjustment.

\section{Relationship between plasma L-PGDS concentrations and clinical indices}

Plasma L-PGDS levels were positively correlated with several parameters (table 2). Plasma L-PGDS also tended to have a positive correlation with morning urinary L-PGDS $(\mathrm{p}=0.07)$. After adjustment for age, sex, BMI, and morning systolic and diastolic blood pressure, plasma L-PGDS levels were only positively correlated with serum creatinine levels $(\beta=0.361 ; p=0.03)$.

\section{Clinical determinants of morning urinary L-PGDS concentrations}

Table 3 shows results of multiple regression analyses to identify those variables (morning systolic and diastolic blood pressure, arousal index, AHI, 3\% ODI, serum HDL-C, plasma adrenalin, plasma noradrenalin and plasma L-PGDS) that could predict morning urinary L-PGDS concentrations. Then, one of the variables that had very strong co-linearity $(r>0.70)$, such as the arousal index, AHI and 3\% ODI, was selected.

In these three models, morning urinary L-PGDS had a significant and independent correlation with the AHI or the $3 \%$ ODI, or the arousal index and serum HDL-C (table 3).

\section{Effects of CPAP treatment on urinary L-PGDS concentrations}

In Japan, patients whose AHI is $\geqslant 20$ can use CPAP under the health insurance system. In this study, 20 permitted patients were investigated (table 4). 12 of the 20 patients had severe OSA.

After 2 days of CPAP treatment, morning urinary L-PGDS concentrations were significantly decreased compared with baseline values (fig. 4a). In contrast, the plasma L-PGDS level, the RH-PAT index (fig. 4b) and the other biomarkers were not significantly changed (table 4). In the 12 patients with severe OSA, morning urinary L-PGDS concentrations were significantly decreased compared with baseline values (591.2 
TABLE 1 Patients' characteristics, polysomnography (PSG) data and laboratory data according to severity of obstructive sleep apnoea (OSA)

\begin{tabular}{|c|c|c|c|c|}
\hline & Control & Moderate OSA & Severe OSA & p-value \\
\hline Subjects & 16 & 25 & 23 & \\
\hline Age years & $47.5(21-76)$ & $55(31-74)$ & $55(27-78)$ & 0.12 \\
\hline Male & $12(75.0)$ & $21(84.0)$ & 21 (91.3) & 0.39 \\
\hline BMI $\mathrm{kg} \cdot \mathrm{m}^{-2}$ & $26.0(20.4-35.8)$ & $24.5(20.2-34.8)$ & $26.6(21.6-39.9)$ & 0.10 \\
\hline Waist circumference $\mathrm{cm}$ & $87(76-118)$ & $90(78-113)$ & $94(82-120)$ & 0.13 \\
\hline Morning systolic BP $\mathrm{mmHg}$ & $114(95-136)$ & 118 (99-139) & $127(96-150)^{\#, 9}$ & 0.003 \\
\hline ESS score & $14(1-24)$ & $12(2-19)$ & $12(4-20)$ & 0.13 \\
\hline \multicolumn{5}{|l|}{ Comorbidity } \\
\hline Hypertension & $3(18.8)$ & $10(40.0)$ & $10(47.8)$ & 0.25 \\
\hline Dyslipidaemia & $9(56.3)$ & $17(68.0)$ & $15(65.2)$ & 0.74 \\
\hline Diabetes mellitus & $2(12.5)$ & $2(8.0)$ & $2(8.7)$ & 0.88 \\
\hline \multicolumn{5}{|l|}{ PSG data } \\
\hline $3 \%$ ODI events $\cdot h^{-1}$ & $5.3(0.5-14.1)$ & $17.4(10.1-27.2)^{\#}$ & $48.0(26.9-86.4)^{\#, \Phi}$ & $<0.0001$ \\
\hline Minimum $\mathrm{SpO}_{2} \%$ & 90.5 (81-97) & $81.5(73-90)^{\#}$ & $75.0(61-86)^{\# \text {. }}$ & $<0.0001$ \\
\hline $\mathrm{SpO}_{2}<90 \% \%$ TST & $0(0-5.0)$ & $2.5(0-8.1)$ & $11.9(1.2-87.7)^{\#, \Phi}$ & $<0.0001$ \\
\hline RH-PAT index & $1.91(1.30-2.87)$ & $2.00(1.32-3.91)$ & $1.65(1.42-3.23)^{\top}$ & 0.02 \\
\hline \multicolumn{5}{|l|}{ Blood } \\
\hline Creatinine $\mathrm{mg} \cdot \mathrm{dL}^{-1}$ & $0.8(0.4-1.0)$ & $0.8(0.6-1.1)$ & $0.8(0.6-1.1)$ & 0.49 \\
\hline $\mathrm{TC} \mathrm{mg} \cdot \mathrm{dL}^{-1}$ & $204(125-241)$ & $197(130-255)$ & $199(141-299)$ & 0.58 \\
\hline $\mathrm{HDL}-\mathrm{C} \mathrm{mg} \cdot \mathrm{dL}^{-1}$ & $51(35-86)$ & 53 (40-93) & $49(30-82)$ & 0.30 \\
\hline $\mathrm{TG} \mathrm{mg} \cdot \mathrm{dL}^{-1}$ & $103(54-245)$ & $100(44-334)$ & $132(70-286)$ & 0.21 \\
\hline $\mathrm{CRP} \mathrm{mg} \cdot \mathrm{dL}^{-1}$ & $0.1(0.0-0.2)$ & $0.1(0.0-0.2)$ & $0.1(0.0-0.5)^{\#, \oplus}$ & 0.004 \\
\hline Glucose $\mathrm{mg} \cdot \mathrm{dL}^{-1}$ & $87(81-114)$ & $95(75-146)$ & $96(85-121)$ & 0.07 \\
\hline Adrenaline $\mathrm{pg} \cdot \mathrm{mL}^{-1}$ & $14(5-31)$ & $16(5-30)$ & $16(5-45)$ & 0.65 \\
\hline
\end{tabular}

Data are presented as n, median (range) or $\mathrm{n}(\%)$, unless otherwise stated. BMI: body mass index; BP: blood pressure; ESS: Epworth Sleepiness Scale; TST: total sleep time; $\mathrm{AHI}$ : apnoea/hypopnoea index; ODI: oxygen desaturation index; $\mathrm{SpO}_{2}$ : arterial oxygen saturation measured by pulse oximetry; RH-PAT: reactive hyperaemia peripheral arterial tone; TC: total cholesterol; HDL-C: high-density lipoprotein cholesterol; TG: triglycerides; CRP: Creactive protein; L-PGDS: lipocalin-type prostaglandin D synthase. ${ }^{\#}: \mathrm{p}<0.0167$ versus control; ${ }^{\circ}: \mathrm{p}<0.0167$ versus moderate 0 OSA.

at baseline versus $317.8 \mathrm{ng} \cdot \mathrm{mg}^{-1}$ of creatinine after 2 days of CPAP; $\mathrm{p}=0.02$ ). Those decreased L-PGDS concentrations reached the levels present in control subjects $(p=0.65)$.

\section{Circadian variations in urinary L-PGDS concentrations}

Circadian variations in urinary L-PGDS concentrations are shown in figure 5a. In 25 patients, a multiple ANOVA with repeated measures revealed a significant time-dependent fluctuation $(\mathrm{p}=0.0002)$, with the highest levels at 14:00 $\mathrm{h}$ and lowest levels at 06:00 h. Compared with the 06:00 h values, urinary L-PGDS concentrations significantly increased at 14:00 $\mathrm{h}(\mathrm{p}<0.0001)$ and 18:00 $\mathrm{h}(\mathrm{p}=0.02)$. Regarding the associations between the AHI and urinary L-PGDS concentrations at each time-point, the 06:00 h values were only significantly positively correlated with $\mathrm{AHI}(\mathrm{r}=0.566 ; \mathrm{p}=0.004)$. In the patients with $\mathrm{AHI} \geqslant 30,06: 00 \mathrm{~h}$ values for urinary L-PGDS were significantly increased compared with patients with AHI $<30$ (AHI $<30(n=13)$ $227 \mathrm{ng} \cdot \mathrm{mg}^{-1}$ of creatinine versus AHI $\geqslant 30(\mathrm{n}=12) 780.3 \mathrm{ng} \cdot \mathrm{mg}^{-1}$ of creatinine; $\left.\mathrm{p}=0.003\right)$ (fig. $5 \mathrm{~b}$ ).

\section{Discussion}

The major findings of our study are that morning urinary L-PGDS concentrations were positively correlated with the severity of OSA as indicated by AHI, 3\% ODI and the arousal index after adjustment for several 

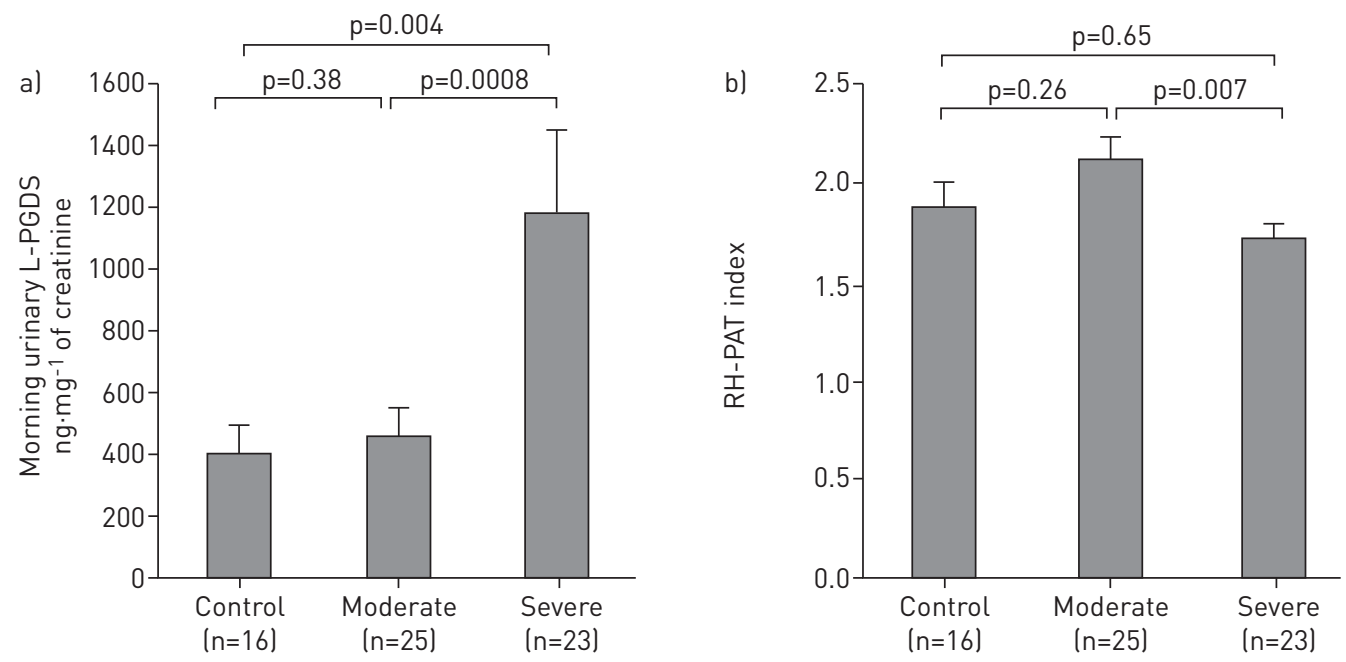

FIGURE 1 Comparison of a) morning urinary lipocalin-type prostaglandin D synthase (L-PGDS) concentrations and b) reactive hyperaemia peripheral arterial tone (RH-PAT) index between controls and moderate and severe obstructive sleep apnoea patients. Data are presented as mean \pm SE.

confounding factors, and that urinary L-PGDS was significantly elevated in patients with severe OSA in comparison with control subjects and those with moderate OSA. Multivariate modelling of L-PGDS determinants revealed that HDL-C and the AHI, 3\% ODI or the arousal index were significantly and independently associated with the morning urinary L-PGDS (table 3). However, it is of interest that urinary L-PGDS levels at 14:00 h were the same in the non-to-moderate and severe OSA patients. Our results also showed that morning urinary L-PGDS concentrations were decreased to control values after only 2 days of CPAP treatment, although the RH-PAT did not change. These results suggest that the urinary L-PGDS concentration might be a moderately useful marker to identify patients with severe OSA.

In the current study, subjects with severe OSA had elevated urinary L-PGDS values and decreased endothelial function determined by the RH-PAT index whereas the relationship between urinary L-PGDS and the RH-PAT index was not significant. Therefore, the combined measurement of endothelial function and L-PGDS may be a useful tool for finding and managing patients with severe OSA.

Prostaglandin systems, including $\mathrm{PGD}_{2}$, are very important in health maintenance and disease prevention, but they are either chemically or metabolically unstable. Therefore, substitutions such as L-PGDS are used as parameters for identifying the functions and effects of prostaglandin systems. Previous studies have demonstrated that L-PGDS levels in serum, cerebrospinal fluid or urine were elevated in patients with cardiovascular, neurological and renal diseases [21, 23, 29, 30]. The elevation in L-PGDS concentration occurs at an early stage of CVD, and the concentrations of serum or urinary L-PGDS were shown to

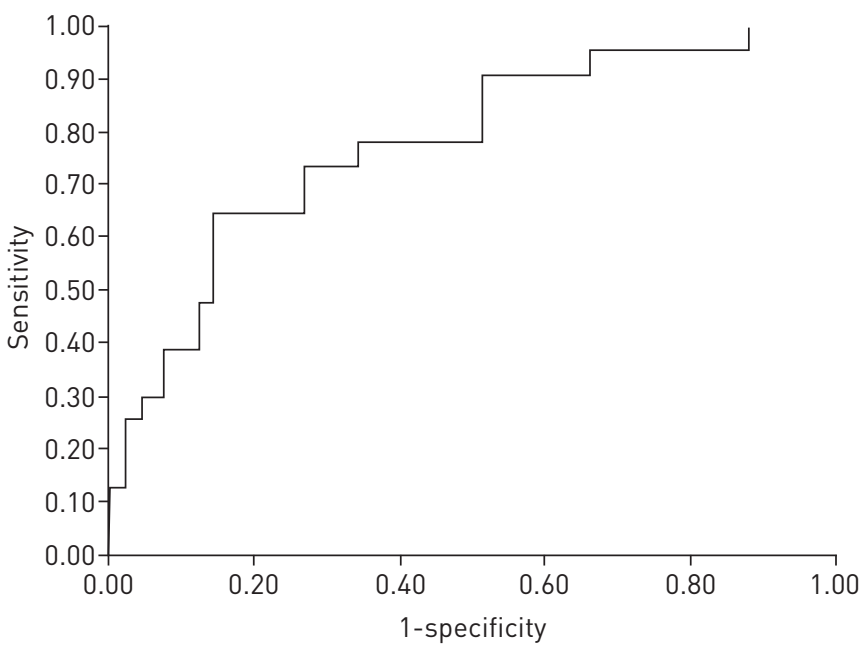

FIGURE 2 Receiver operating characteristic curve analysis to assess the diagnostic validity of morning urinary lipocalin-type prostaglandin D synthase concentrations to detect severe obstructive sleep apnoea. 
TABLE 2 Associations of lipocalin-type prostaglandin D synthase (L-PGDS) level and reactive hyperaemia peripheral arterial tone (RH-PAT) index with patients' characteristics, polysomnography data and biomarkers

\begin{tabular}{|c|c|c|c|c|c|c|}
\hline & \multicolumn{2}{|c|}{$\begin{array}{l}\text { Morning urinary L-PGDS } \\
\mathrm{ng} \cdot \mathrm{mg}^{-1} \text { of creatinine }\end{array}$} & \multicolumn{2}{|c|}{ Plasma L-PGDS $\mathrm{ng} \cdot \mathrm{mL}^{-1}$} & \multicolumn{2}{|c|}{ RH-PAT index } \\
\hline & $\mathbf{r}$ & $p$-value & $\mathbf{r}$ & p-value & $\mathbf{r}$ & p-value \\
\hline Age years & 0.164 & 0.20 & 0.362 & 0.003 & 0.074 & 0.56 \\
\hline Male & -0.013 & 0.92 & 0.048 & 0.71 & -0.160 & 0.21 \\
\hline $\mathrm{BMI} \mathrm{kg} \cdot \mathrm{m}^{-2}$ & -0.060 & 0.64 & -0.132 & 0.31 & -0.135 & 0.29 \\
\hline Morning diastolic BP $\mathrm{mmHg}$ & 0.323 & $0.009 *$ & 0.274 & $0.03^{*}$ & 0.001 & 0.99 \\
\hline ESS & -0.139 & 0.27 & -0.035 & 0.78 & 0.075 & 0.56 \\
\hline Arousal events $\cdot h^{-1}$ & 0.472 & $<0.0001 *$ & 0.220 & 0.08 & -0.268 & $0.03^{*}$ \\
\hline AHI events $\cdot h^{-1}$ & 0.426 & $0.0005^{*}$ & 0.180 & 0.16 & -0.241 & 0.06 \\
\hline $3 \%$ ODI events $\cdot h^{-1}$ & 0.384 & $0.002^{*}$ & 0.173 & 0.18 & -0.244 & 0.054 \\
\hline Minimum $\mathrm{SpO}_{2} \%$ & -0.112 & 0.38 & 0.099 & 0.44 & 0.046 & 0.72 \\
\hline $\mathrm{HDL}-\mathrm{C} \mathrm{mg} \cdot \mathrm{dL}^{-1}$ & 0.214 & 0.09 & 0.045 & 0.73 & 0.079 & 0.54 \\
\hline$T G \mathrm{mg} \cdot \mathrm{dL}^{-1}$ & -0.067 & 0.60 & 0.170 & 0.18 & 0.134 & 0.30 \\
\hline CRP $\mathrm{mg} \cdot \mathrm{dL}^{-1}$ & 0.071 & 0.58 & 0.083 & 0.52 & -0.132 & 0.30 \\
\hline Glucose $\mathrm{mg} \cdot \mathrm{dL}^{-1}$ & 0.158 & 0.22 & -0.161 & 0.21 & -0.143 & 0.27 \\
\hline Adrenaline $\mathrm{pg} \cdot \mathrm{mL}^{-1}$ & 0.310 & $0.02^{*}$ & 0.220 & 0.10 & 0.018 & 0.89 \\
\hline Noradrenaline $\mathrm{pg} \cdot \mathrm{mL}^{-1}$ & 0.329 & $0.008^{*}$ & 0.149 & 0.25 & 0.128 & 0.32 \\
\hline Plasma L-PGDS $\mathrm{ng} \cdot \mathrm{mL}^{-1}$ & 0.228 & 0.07 & & & 0.030 & 0.82 \\
\hline $\begin{array}{l}\text { Morning urinary L-PGDS } \\
\mathrm{ng} \cdot \mathrm{mg}^{-1} \text { of creatinine }\end{array}$ & & & 0.228 & 0.07 & -0.068 & 0.59 \\
\hline $\begin{array}{l}\text { Night urinary L-PGDS } \mathrm{ng} \cdot \mathrm{mg}^{-1} \\
\text { of creatinine }\end{array}$ & 0.868 & $<0.0001^{*}$ & 0.090 & 0.51 & 0.050 & 0.71 \\
\hline
\end{tabular}

BMI: body mass index; BP: blood pressure; ESS: Epworth Sleepiness Scale; AHI: apnoea/hypopnoea index; ODI: oxygen desaturation index; SpO ${ }_{2}$ : arterial oxygen saturation measured by pulse oximetry; TST: total sleep time; TC: total cholesterol; HDL-C: high-density lipoprotein cholesterol; TG: triglycerides; CRP: C-reactive protein. ${ }^{*}: \mathrm{p}<0.05$.

increase when diseases such as coronary heart disease or type 2 DM worsen [21-23], although the activity of L-PGDS is not well known. OSA induces multi-organ damage such as hypersomnolence and CVD. Therefore, it is supposed that L-PGDS could have significant associations with the pathophysiology and severity of OSA. In addition, a urinary biomarker is attractive because urine is easily obtained and the results of urine testing are easy to follow. Although our present data might not be definitive because of the small sample size, the information gained from this study appears to be of interest and the topic warrants further study.

It has been said that serum L-PGDS levels increase with age and are higher in males than in females [19], while urinary L-PGDS was also shown to be higher in males than in females and is weakly correlated with age in female subjects [22]. In that study [22], control subjects were slightly younger, but not with significance, and those in the moderate and severe OSA groups were almost the same age as those in the present study (table 1). In addition, morning urinary L-PGDS values were still correlated with the severity of OSA after adjustment for BMI, age and sex. Therefore, BMI, sex and age could not be considered to have significant effects on our results. Although L-PGDS concentrations were shown to be increased in essential hypertension [21], morning urinary L-PGDS values were still correlated with the severity of OSA after adjustment for blood pressure. The BMI in individuals with severe OSA was slightly but not significantly higher than in the other groups. It was reported that the BMI [21] was not associated with elevations in blood or urinary L-PGDS. In addition to the fact that morning urinary L-PGDS levels were significantly higher after adjustment for BMI, morning urinary L-PGDS concentrations were also significantly decreased after 2 days of CPAP, which is a specific treatment for OSA, although other factors remained the same. Furthermore, from the viewpoint of circadian measurements, morning urine L-PGDS levels were higher in 


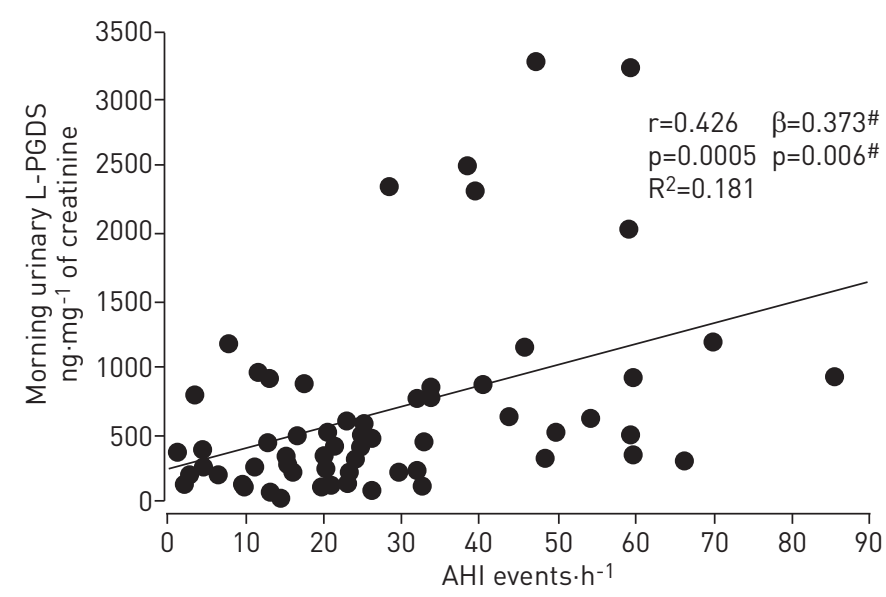

FIGURE 3 Relationship between apnoea/hypopnoea index (AHI) and morning urinary lipocalintype prostaglandin D synthase (LPGDS) concentrations. " : values after adjustment for age, sex, body mass index, and morning systolic and diastolic blood pressure.

patients with severe OSA than in the other subjects, while values at 14:00 h were the same. These findings indicate that L-PGDS is related to OSA independently, and is especially related to severe OSA.

Several causes for elevated L-PGDS concentrations in OSA have been considered, especially in severe OSA. First, intermittent hypoxia induces COX2 expression and activity, which in turn accelerate arachnoid acid metabolism to $\mathrm{PGH}_{2}$ and $\mathrm{PGE}_{2}[31,32]$. HAN et al. [33] noted that the expression levels of L-PGDS mRNA and protein in the heart of C57BL/6 mice were significantly increased after 14 days of hypoxia $(10 \%$ oxygen) compared with mice kept under normoxia. OSA is characterised by repeated episodes of oxygen desaturation; however, in OSA there is also sustained hypoxaemia, which is expressed by arterial oxygen saturation measured by pulse oximetry $<90 \%$ ( $\%$ of total sleep time). Thus, both sustained and intermittent hypoxaemia could possibly increase urinary L-PGDS. Secondly, hypoxia causes pulmonary vasoconstriction and increases pulmonary artery pressure [34]. It has been reported that fluid shear stress increases the expression of L-PGDS in vascular endothelial cells $[35,36]$ and that urinary $\mathrm{PGD}_{2}$ metabolites were increased in primary pulmonary hypertension [37]. SEMENZA [38] reported that intermittent hypoxaemia induced systemic hypertension and that sustained hypoxaemia induced pulmonary hypertension. Both systemic and pulmonary hypertension induced by OSA might increase L-PGDS values [21, 37].

It has been shown that sleep fragmentation and arousals caused by sleep apnoea induce an elevation of sympathetic activation [39]. If the severity of OSA might be associated with urinary or plasma L-PGDS concentrations, we thought that it would be important to determine whether there is a significant relationship between sympathetic activation and urinary or plasma L-PGDS. Therefore, we wanted to measure urinary catecholamine concentrations as indicators of sympathetic activity. However, it was

\begin{tabular}{|c|c|c|c|c|}
\hline & $\beta$ & $r$ & p-value & $\mathbf{R}^{2} \%$ \\
\hline \multicolumn{5}{|l|}{ Model 1} \\
\hline $\mathrm{AHI}$ events $\cdot \mathrm{h}^{-1}$ & 0.326 & 0.426 & 0.02 & 13.9 \\
\hline $\mathrm{HDL}-\mathrm{C} \mathrm{mg} \cdot \mathrm{dL}^{-1}$ & 0.297 & 0.214 & 0.02 & 6.4 \\
\hline Cumulative $\mathrm{R}^{2}$ & & & & 20.3 \\
\hline \multicolumn{5}{|l|}{ Model 2} \\
\hline Arousal, events $\cdot \mathrm{h}^{-1}$ & 0.411 & 0.472 & 0.001 & 19.4 \\
\hline $\mathrm{HDL}-\mathrm{C} \mathrm{mg} \cdot \mathrm{dL}^{-1}$ & 0.290 & 0.214 & 0.01 & 6.2 \\
\hline Cumulative $\mathrm{R}^{2}$ & & & & 25.6 \\
\hline \multicolumn{5}{|l|}{ Model 3} \\
\hline $3 \%$ ODI events $\cdot \mathrm{h}^{-1}$ & 0.291 & 0.384 & 0.03 & 11.2 \\
\hline $\mathrm{HDL}-\mathrm{C} \mathrm{mg} \cdot \mathrm{dL}^{-1}$ & 0.280 & 0.214 & 0.02 & 6.0 \\
\hline Cumulative $\mathrm{R}^{2}$ & & & & 17.2 \\
\hline
\end{tabular}

$\beta$ : standard regression coefficient; $r$ : correlation coefficient; $R^{2}$ : contribution rate; $A H I$ : apnoea/hypopnoea index; HDL-C: high-density lipoprotein cholesterol; ODI: oxygen desaturation index. 
TABLE 4 Effects of continuous positive airway pressure (CPAP) on the reactive hyperaemia peripheral arterial tone (RH-PAT) index, various parameters and lipocalin-type prostaglandin D synthase (L-PGDS)

\begin{tabular}{|c|c|c|c|}
\hline & Before CPAP & After 2 days of CPAP & p-value \\
\hline BMI & $25.2(21.0-39.9)$ & $24.8(20.8-39.9)$ & 0.11 \\
\hline Morning systolic BP mmHg & $126(102-138)$ & $121(103-138)$ & 0.15 \\
\hline Arousal index events $\cdot \mathrm{h}^{-1}$ & $31.6(10.8-54.8)$ & $16.8(8.6-42.8)$ & 0.0005 \\
\hline AHI events $\cdot h^{-1}$ & $33.6(20.3-59.6)$ & $4.4(0-8.8)$ & $<0.0001$ \\
\hline $3 \%$ ODI events $\cdot \mathrm{h}^{-1}$ & $27.9(16.2-61.3)$ & $3.5(0-7.6)$ & $<0.0001$ \\
\hline Creatinine $\mathrm{mg} \cdot \mathrm{dL}^{-1}$ & $0.8(0.6-1.0)$ & $0.8(0.6-1.0)$ & 0.41 \\
\hline $\mathrm{TC} \mathrm{mg} \cdot \mathrm{dL}^{-1}$ & $189(130-299)$ & 184 (132-293) & 0.78 \\
\hline $\mathrm{HDL}-\mathrm{C} \mathrm{mg} \cdot \mathrm{dL}^{-1}$ & $53(41-66)$ & $52(38-68)$ & 0.13 \\
\hline$T G \mathrm{mg} \cdot \mathrm{dL}^{-1}$ & $95(44-215)$ & $98(58-304)$ & 0.30 \\
\hline $\mathrm{CRP} \mathrm{mg} \cdot \mathrm{dL}^{-1}$ & $0.1(0.0-0.5)$ & $0.0(0.0-0.4)$ & 0.07 \\
\hline Glucose $\mathrm{mg} \cdot \mathrm{dL}^{-1}$ & $96(75-133)$ & $96(83-140)$ & 0.23 \\
\hline $\begin{array}{l}\text { Morning L-PGDS ng. } \mathrm{mg}^{-1} \text { of } \\
\text { creatinine }\end{array}$ & $591.2(227-3274.1)$ & $317.8(130.3-1587.6)$ & 0.007 \\
\hline
\end{tabular}

Data are presented as median (range), unless otherwise stated. BMI: body mass index; BP: blood pressure; AHI: apnoea/hypopnoea index; ODI: oxygen desaturation index; TC: total cholesterol; HDL-C: high-density lipoprotein cholesterol; TG: triglycerides; CRP: C-reactive protein.

difficult to obtain urine in which L-PGDS and catecholamine levels could be measured at the same time. Therefore, even though we knew that plasma catecholamine levels were unstable and easily variable, the usefulness of which was difficult to understand, we measured plasma catecholamine levels. In the present study, urinary L-PGDS concentrations were also correlated with plasma adrenaline and noradrenaline. Therefore, sympathetic overactivity in OSA $[40,41]$ might also induce increases in urinary L-PGDS. The relationship between urinary catecholamine and L-PGDS should be studied in the future. We also found that HDL-C was a significant determinant of morning urinary L-PGDS. MiwA et al. [42] reported that
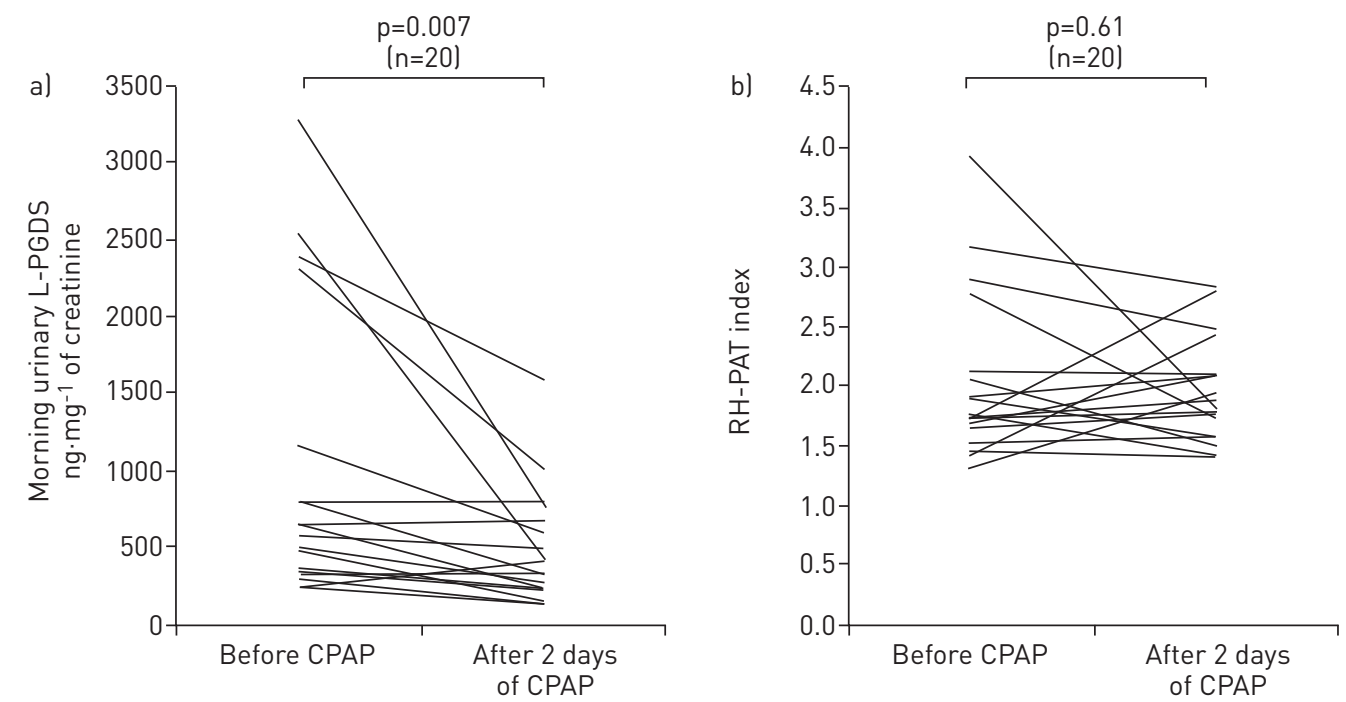

FIGURE 4 Change in a) morning urinary lipocalin-type prostaglandin D synthase (L-PGDS) concentrations and b) reactive hyperaemia peripheral arterial tone (RH-PAT) index before and after 2 days of continuous positive airway pressure (CPAP). Individual data are presented. 

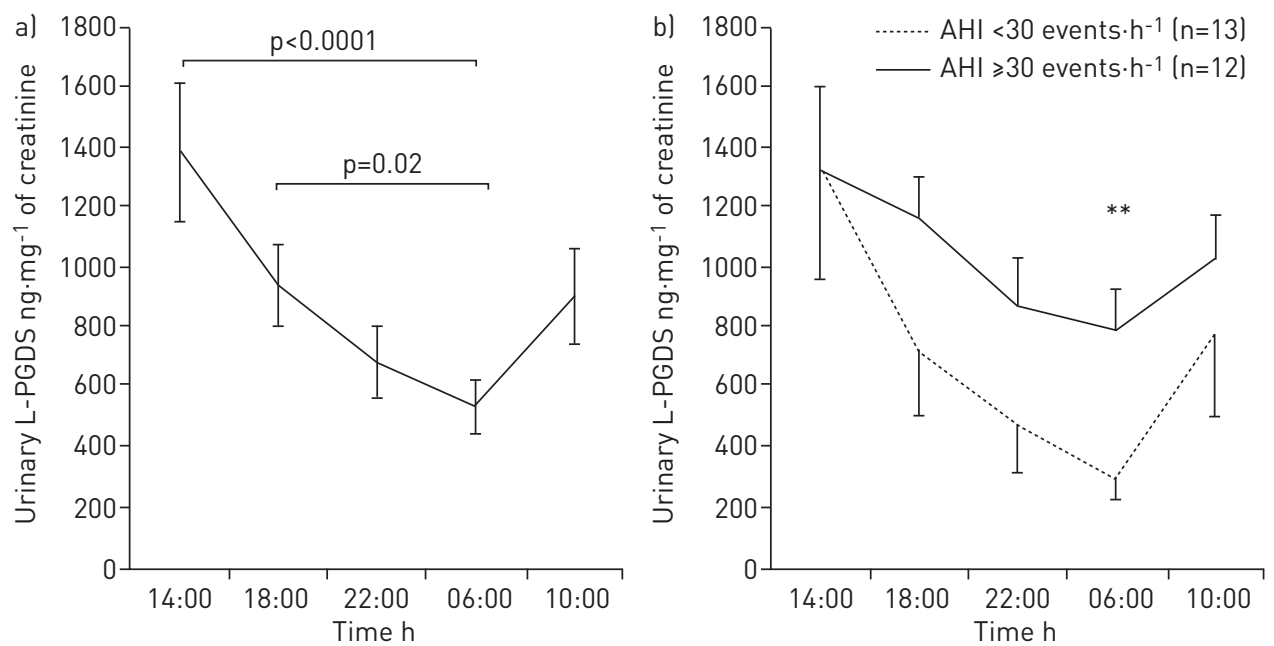

FIGURE 5 a) Urinary lipocalin-type prostaglandin D synthase (L-PGDS) concentrations in 25 study patients at 4-h intervals within a day (except midnight). b) shows the comparison of circadian variations in urinary L-PGDS between patients with apnoea/hypopnoea index (AHI) $\geqslant 30$ events $\cdot \mathrm{h}^{-1}(\mathrm{n}=12)$ and patients with AHI $<30$ events $\cdot \mathrm{h}^{-1}(\mathrm{n}=13)$. Data are shown as mean \pm SE. ${ }^{* *}: \mathrm{p}<0.01$ between patients with AHI $\geqslant 30$ events $\cdot \mathrm{h}^{-1}$ and those with AHI $<30$ events $\cdot \mathrm{h}^{-1}$.

L-PGDS played a role in lipid transport. Therefore, HDL-C might be a significant factor along with the arousal index or the AHI in determining the urinary L-PGDS levels.

The present study is the first to demonstrate the circadian variations in urinary L-PGDS concentrations in OSA. Urinary L-PGDS values in both severe and non-to-moderate OSA were highest and at the same levels at 14:00 h, with the lowest values at 06:00 $\mathrm{h}$ in both groups. However, only 06:00 $\mathrm{h}$ urinary L-PGDS values correlated significantly with the AHI and were significantly higher in those with severe OSA compared with subjects with AHI $<30$ events $\cdot \mathrm{h}^{-1}$. In the current study, we showed that morning urinary L-PGDS concentrations were positively correlated with the AHI, 3\% ODI and arousal index. Through the circadian change in L-PGDS, we propose that L-PGDS might be a stress marker that increases during daytime and decreases during sleep, whereas the decrease in L-PGDS levels in OSA, especially severe OSA, during sleep is attenuated because intermittent or sustained hypoxia, sleep fragmentation and arousals caused by OSA lead to increased stress, including oxidative stress and sympathetic activation during sleep [39]. Additionally, occurrences of CVDs peak from morning to noon, while OSA patients have an increased risk of myocardial infarction between 00:00 $\mathrm{h}$ and 06:00 h compared with non-OSA patients [43]. These circadian rhythms mimic the pattern of urinary L-PGDS in this study (fig. 4a).

In the current study, we did not detect a significant relationship between plasma L-PGDS and the AHI, whereas morning urinary L-PGDS levels were significantly positively correlated with the AHI. Although the reason for this discrepancy is unclear, HIRAWA et al. [44] reported that urinary protein excretion in the early stage of DM was correlated with urinary L-PGDS excretion, but not with plasma L-PGDS levels. In addition, serum L-PGDS levels were not shown to be associated with the AHI [25]. The influence of OSA, such as intermittent hypoxaemia, might have a significant effect on the renal system, which induced the differences in values between plasma and urinary L-PGDS. The differences in L-PGDS levels between plasma and urine should be studied in animal models. Furthermore, we did not collect peripheral blood samples at 22:00 $\mathrm{h}$ in the current study. The night plasma L-PGDS concentrations or the differences between morning and night plasma L-PGDS may contribute to elucidating the relation between plasma L-PGDS and OSA.

This study had some limitations. First, the sample size was small. However, the differences in the urinary L-PGDS values between control, moderate and severe OSA patients were large and, therefore, the results could be considered significant and definitive. Secondly, it is unclear whether the effect of CPAP on the $\mathrm{PGD}_{2}$ system would persist over the long term. A long-term prospective study is needed to clarify this issue. Thirdly, we could not conduct a comparison between CPAP users and sham CPAP users. A future study that makes comparisons between CPAP users and sham CPAP users is warranted. Fourthly, we used spot urine samples for measurement of L-PGDS. There is a possibility that several factors, such as reabsorption at tubules and physical activity, influenced the urinary L-PGDS concentrations. However, use of overnight spot urine for measurement of L-PGDS has been validated because of the correlation between L-PGDS 
values of overnight urine and urine collected over $24 \mathrm{~h}$ [44]. Therefore, we believe overnight spot urine sampling is sufficient to evaluate the role of L-PGDS in OSA.

In conclusion, based on our results, in addition to circadian data, urinary L-PGDS might be a moderately useful marker for severe OSA. From these preliminary data, urine L-PGDS measurement may be a simple and cost-effective method to screen for and manage severe OSA. This method should be tested in unselected samples in the future because it is often difficult, costly and time consuming to find patients with OSA, while the number of OSA patients who should be treated is large.

\section{Acknowledgements}

The authors thank O. Hayaishi (Dept Molecular Behavioural Biology, Osaka Bioscience Institute, Osaka, Japan) for his kind comments and suggestions for this manuscript. We thank M. Azuma, K. Aihara, K. Tanizawa and T. Handa (Dept of Respiratory Medicine, Graduate School of Medicine, Kyoto University, Kyoto, Japan), K. Yamammoto (Dept of Allergy and Rheumatology, Graduate School of Medicine, Kyoto University) and T. Kimura (Dept of Cardiovascular Medicine, Graduate School of Medicine, Kyoto University) for valuable advice in manuscript preparation. We also thank T. Toki, S. Tamura and N. Kimura (Dept of Respiratory Care and Sleep Control Medicine, Graduate School of Medicine, Kyoto University) for their support for this clinical research. In addition, we thank K. Ueda, Y. Yamanishi, N. Susukida and N. Matsuura (Dept of Clinical Laboratory, Graduate School of Medicine, Kyoto University) for their contribution in analysing polysomnographic data.

\section{References}

1 Douglas NJ. Sleep apnea. In: Longo DL, Fauci AS, Kasper DL, et al., eds. Harrison's Principle of Internal Medicine. 17th Edn. New York, McGraw-Hill, 2008; pp. 1665-1668.

2 Ogorochi T, Narumiya S, Mizuno N, et al. Regional distribution of prostaglandins $\mathrm{D}_{2}, \mathrm{E}_{2}$, and $\mathrm{F}_{2 \alpha}$ and related enzymes in postmortem human brain. J Neurochem 1984; 43: 71-82.

3 Whittle BJ, Moncada S, Mullane K, et al. Platelet and cardiovascular activity of the hydantoin BW245C, a potent prostaglandin analogue. Prostaglandins 1983; 25: 205-223.

4 Narumiya $\mathrm{S}$, Toda N. Different responsiveness of prostaglandin $\mathrm{D}_{2}$-sensitive systems to prostaglandin $\mathrm{D}_{2}$ and its analogues. Br J Pharmacol 1985; 85: 367-375.

5 Casteleijn E, Kuiper J, Van Rooij HC, et al. Prostaglandin $\mathrm{D}_{2}$ mediates the stimulation of glycogenolysis in the liver by phorbol ester. Biochem J 1988; 250: 77-80.

6 Darius H, Michael-Hepp J, Thierauch KH, et al. Inhibition of human platelets and polymorphonuclear neutrophils by the potent and metabolically stable prostaglandin $\mathrm{D}_{2}$ analog ZK 118.182. Eur J Pharmacol 1994; 258: 207-213.

7 Matsugi T, Kageyama M, Nishimura $\mathrm{K}$, et al. Selective prostaglandin $\mathrm{D}_{2}$ receptor stimulation elicits ocular hypotensive effects in rabbits and cats. Eur J Pharmacol 1995; 275: 245-250.

8 Matsuoka T, Hirata M, Tanaka H, et al. Prostaglandin $\mathrm{D}_{2}$ as a mediator of allergic asthma. Science 2000; 287: 2013-2017.

9 Eguchi N, Minami T, Shirafuji N, et al. Lack of tactile pain (allodynia) in lipocalin-type prostaglandin D synthasedeficient mice. Proc Natl Acad Sci USA 1999; 96: 726-730.

10 Urade Y, Hayaishi O. Prostaglandin $\mathrm{D}_{2}$ and sleep regulation. Biochim Biophys Acta 1999; 1436: 606-615.

11 Mizoguchi A, Eguchi N, Kimura K, et al. Dominant localization of prostaglandin D receptors on arachnoid trabecular cells in mouse basal forebrain and their involvement in the regulation of non-rapid eye movement sleep. Proc Natl Acad Sci USA 2001; 98: 11674-11679.

12 Hayaishi O. Molecular genetic studies on sleep-wake regulation, with special emphasis on the prostaglandin $\mathrm{D}_{2}$ system. J Appl Physiol 2002; 92: 863-868.

13 Matsuoka T, Narumiya S. The roles of prostanoids in infection and sickness behaviors. J Infect Chemother 2008; 14: 270-278.

14 Urade Y, Fujimoto N, Hayaishi O. Purification and characterization of rat brain prostaglandin D synthetase. J Biol Chem 1985; 260: 12410-12415.

15 Oda H, Eguchi N, Urade Y. Quantitative sandwich enzyme-linked immunosorbent assay for human secretory prostagrandin D synthase ( $\beta$-trace). Proc Japan Acad 1996; 72: 108-111.

16 Li W, Mase M, Inui T, et al. Pharmacokinetics of recombinant human lipocalin-type prostaglandin D synthase/ beta-trace in canine. Neurosci Res 2008; 61: 289-293.

17 Eguchi Y, Eguchi N, Oda H, et al. Expression of lipocalin-type prostaglandin D synthase (beta-trace) in human heart and its accumulation in the coronary circulation of angina patients. Proc Natl Acad Sci USA 1997; 94: 14689-14694.

18 Oda H, Shiina Y, Seiki K, et al. Development and evaluation of a practical ELISA for human urinary lipocalin-type prostaglandin D synthase. Clin Chem 2002; 48: 1445-1453.

19 Miwa Y, Oda H, Shiina Y, et al. Association of serum lipocalin-type prostaglandin D synthase levels with subclinical atherosclerosis in untreated asymptomatic subjects. Hypertens Res 2008; 31: 1931-1939.

20 Urade Y, Ujihara M, Horiguchi Y, et al. Mast cells contain spleen-type prostaglandin D synthetase. J Biol Chem 1990; 265: 371-375.

21 Hirawa N, Uehara Y, Yamakado M, et al. Lipocalin-type prostaglandin d synthase in essential hypertension. Hypertension 2002; 39: 449-454.

22 Uehara Y, Makino H, Seiki K, et al. Urinary excretions of lipocalin-type prostaglandin D synthase predict renal injury in type-2 diabetes: a cross-sectional and prospective multicentre study. Nephrol Dial Transplant 2009; 24: 475-482.

23 Inoue $\mathrm{T}$, Eguchi $\mathrm{Y}$, Matsumoto $\mathrm{T}$, et al. Lipocalin-type prostaglandin $\mathrm{D}$ synthase is a powerful biomarker for severity of stable coronary artery disease. Atherosclerosis 2008; 201: 385-391.

24 Somers VK, White DP, Amin R, et al. Sleep apnea and cardiovascular disease: an American Heart Association/ American College Of Cardiology Foundation Scientific Statement from the American Heart Association Council 
for High Blood Pressure Research Professional Education Committee, Council on Clinical Cardiology, Stroke Council, and Council On Cardiovascular Nursing. In collaboration with the National Heart, Lung, and Blood Institute National Center on Sleep Disorders Research (National Institutes of Health). Circulation 2008; 118 : $1080-1111$

25 Barceló A, de la Peña M, Barbé F, et al. Prostaglandin D synthase (beta trace) levels in sleep apnea patients with and without sleepiness. Sleep Med 2007; 8: 509-511.

26 Teramoto T, Sasaki J, Ueshima H, et al. Diagnostic criteria for dyslipidemia. Executive summary of Japan Atherosclerosis Society (JAS) guideline for diagnosis and prevention of atherosclerotic cardiovascular diseases for Japanese. J Atheroscler Thromb 2007; 14: 155-158.

27 Bonetti PO, Barsness GW, Keelan PC, et al. Enhanced external counterpulsation improves endothelial function in patients with symptomatic coronary artery disease. J Am Coll Cardiol 2003; 41: 1761-1768.

28 Itzhaki S, Lavie L, Pillar G, et al. Endothelial dysfunction in obstructive sleep apnea measured by peripheral arterial tone response in the finger to reactive hyperemia. Sleep 2005; 28: 594-600.

29 Melegos DN, Freedman MS, Diamandis EP. Prostaglandin D synthase concentration in cerebrospinal fluid and serum of patients with neurological disorders. Prostaglandins 1997; 54: 463-474.

30 Melegos DN, Grass L, Pierratos A, et al. Highly elevated levels of prostaglandin D synthase in the serum of patients with renal failure. Urology 1999; 53: 32-37.

31 Gozal D, Kheirandish L. Sleepiness and neurodegeneration in sleep-disordered breathing: convergence of signaling cascades. Am J Respir Crit Care Med 2005; 171: 1325-1327.

32 Li RC, Row BW, Gozal E, et al. Cyclooxygenase 2 and intermittent hypoxia-induced spatial deficits in the rat. Am J Respir Crit Care Med 2003; 168: 469-475.

33 Han F, Takeda K, Ishikawa K, et al. Induction of lipocalin-type prostaglandin D synthase in mouse heart under hypoxemia. Biochem Biophys Res Commun 2009; 385: 449-453.

34 Tilkian AG, Guilleminault C, Schroeder JS, et al. Hemodynamics in sleep-induced apnea. Studies during wakefulness and sleep. Ann Intern Med 1976; 85: 714-719.

35 Taba $\mathrm{Y}$, Sasaguri T, Miyagi M, et al. Fluid shear stress induces lipocalin-type prostaglandin $\mathrm{D}_{2}$ synthase expression in vascular endothelial cells. Circ Res 2000; 86: 967-973.

36 Miyagi M, Miwa Y, Takahashi-Yanaga F, et al. Activator protein-1 mediates shear stress-induced prostaglandin D synthase gene expression in vascular endothelial cells. Arterioscler Thromb Vasc Biol 2005; 25: 970-975.

37 Robbins IM, Barst RJ, Rubin LJ, et al. Increased levels of prostaglandin $\mathrm{D}_{2}$ suggest macrophage activation in patients with primary pulmonary hypertension. Chest 2001; 120: 1639-1644.

38 Semenza GL. Regulation of oxygen homeostasis by hypoxia-inducible factor 1. Physiology (Bethesda) 2009; 24: 97-106.

39 Arnardottir ES, Mackiewicz M, Gislason T, et al. Molecular signatures of obstructive sleep apnea in adults: a review and perspective. Sleep 2009; 32: 447-470.

40 Narkiewicz K, van de Borne PJ, Cooley RL, et al. Sympathetic activity in obese subjects with and without obstructive sleep apnea. Circulation 1998; 98: 772-776.

41 Ziegler MG, Mills PJ, Loredo JS, et al. Effect of continuous positive airway pressure and placebo treatment on sympathetic nervous activity in patients with obstructive sleep apnea. Chest 2001; 120: 887-893.

42 Miwa Y, Takiuchi S, Kamide K, et al. Identification of gene polymorphism in lipocalin-type prostaglandin D synthase and its association with carotid atherosclerosis in Japanese hypertensive patients. Biochem Biophys Res Commun 2004; 322: 428-433.

43 Kuniyoshi FH, Garcia-Touchard A, Gami AS, et al. Day-night variation of acute myocardial infarction in obstructive sleep apnea. J Am Coll Cardiol 2008; 52: 343-346.

44 Hirawa N, Uehara Y, Ikeda $\mathrm{T}$, et al. Urinary prostaglandin D synthase (beta-trace) excretion increases in the early stage of diabetes mellitus. Nephron 2001; 87: 321-327. 